\title{
Can supernova neutrino nucleosynthesis constrain neutrino oscillation parameters?
}

Takashi Yoshida*

National Astronomical Observatory of Japan

E-mail: takashi.yoshida@nao.ac.jp

\section{Toshitaka Kajino}

National Astronomical Observatory of Japan and the Graduate University for Advanced Studies Department of Astronomy, University of Tokyo

\section{Hidekazu Yokomakura, Keiichi Kimura}

Department of Physics, Nagoya University

\section{Akira Takamura}

Department of Mathematics, Toyota National College of Technology

\section{Dieter H. Hartmann}

Department of Physics and Astronomy, Clemson University

Recent neutrino experiment confirmed that neutrinos have finite masses and that neutrino oscillations occur. Supernova neutrinos also change their flavors propagating in the stellar material surrounding a proto-neutron star and, accordingly, change their spectral energy distributions. The nucleosynthesis of light elements such as ${ }^{7} \mathrm{Li}$ and ${ }^{11} \mathrm{~B}$ is significantly enhanced through the $v$ process in supernova explosions. Yields depend on the energy spectra of the neutrinos emitted from the proto-neutron star, so that neutrino oscillations could be crucial for the estimated yields of ${ }^{7} \mathrm{Li},{ }^{11} \mathrm{~B}$, and other isotopes produced in supernovae. We evaluate the ${ }^{7} \mathrm{Li}$ and ${ }^{11} \mathrm{~B}$ yields in supernovae taking into account changes of the neutrino energy spectra due to flavor oscillations assuming large mixing angle solutions. We investigate their dependence on key parameters, such as mass hierarchies and the mixing angle $\theta_{13}$. In the case of a normal mass hierarchy and an adiabatic resonance of 13 -mixing, the ${ }^{7} \mathrm{Li}$ yield is larger than that without neutrino oscillations by a factor of $\sim 2$. For other parameter cases and for the ${ }^{11} \mathrm{~B}$ yield in general, the enhancement is smaller. The increase of the ${ }^{7} \mathrm{Li} /{ }^{11} \mathrm{~B}$ ratio is demonstrated for a normal mass hierarchy and a relatively large $\sin ^{2} 2 \theta_{13}$, where 13 -mixing resonance is adiabatic and $\sin ^{2} 2 \theta_{13}<0.1$. Thus, the detection of ${ }^{7} \mathrm{Li} /{ }^{11} \mathrm{~B}$ enhancements in stars showing signs of supernova enrichment suggest a normal mass hierarchy and relatively large value of $\sin ^{2} 2 \theta_{13}$.

International Symposium on Nuclear Astrophysics - Nuclei in the Cosmos - IX

June 25-30 2006

CERN, Geneva, Switzerland

\footnotetext{
* Speaker.
} 


\section{Introduction}

Recent observations of solar, atmospheric, and reactor neutrinos confirmed the existence of flavor oscillations (for a recent review see [1]). These experiments have precisely determined most of the neutrino oscillation parameters, such as the squared-mass differences and mixing angles. However, definite constraints on two oscillation parameters have not been obtained; the mass hierarchy has not been determined and only upper limits of the mixing angle $\theta_{13}$ have been obtained.

During a supernova (SN) explosion, a very large neutrino luminosity emanates from the cooling proto-neutron star (PNS). The resulting large neutrino irradiation of the stellar layers above the PNS induces significant modifications of the isotopic abundance patterns, which is referred to as the $v$-process. This process plays an important role for the production of, amongst several, the light elements ${ }^{7} \mathrm{Li}$ and ${ }^{11} \mathrm{~B}[2,3,4]$. On the other hand, it has been shown that the neutrinos change their flavors in the $\mathrm{O} / \mathrm{C}$ and He-rich layers and that this flavor change strongly depends on the mass hierarchy and the mixing angle $\theta_{13}$ (e.g., [5]). This implies that the neutrino energy spectra change in these layers and that the extent of the spectral modifications depends on these two parameters. We have shown in [4] that the ${ }^{7} \mathrm{Li}$ and ${ }^{11} \mathrm{~B}$ yields depend on the neutrino energy spectra. If the $v_{e}$ and $\bar{v}_{e}$ spectra change, the ${ }^{7} \mathrm{Li}$ and ${ }^{11} \mathrm{~B}$ yields are affected because of the contribution from charged-current $v$-process reactions. In this study, we investigate the effects of oscillations on ${ }^{7} \mathrm{Li}$ and ${ }^{11} \mathrm{~B}$ production, and evaluate the dependence of these yields on assumed mass hierarchies and the mixing angle $\theta_{13}$. We further discuss the possibility for constraining oscillation parameters with light element synthesis in supernovae.

\section{Supernova nucleosynthesis model and neutrino parameters}

We adopt the same stellar model as that described in [3, 4, 6, 7]. The SN progenitor is a 16.2 $M_{\odot}$ star [8], and the explosion is followed numerically with a piecewise parabolic method [9]. We set the explosion energy and the mass cut to be $1 \times 10^{51} \mathrm{ergs}$ and $1.61 M_{\odot}$, respectively. Detailed explosive nucleosynthesis is calculated by postprocessing using a nuclear reaction network including 291 species of nuclei [3].

We adopt the following assumptions regarding neutrinos emitted from the PNS's neutrino sphere. The luminosity decays exponentially with a decay time scale of $3 \mathrm{~s}$ and is equally partitioned by each flavor. The neutrino energy spectra obey Fermi-Dirac distributions. As the standard model of this study, the total neutrino energy and the neutrino temperatures are set to be $E_{v}=3 \times 10^{53} \mathrm{ergs}, T_{v_{e}}=3.2 \mathrm{MeV}, T_{\bar{v}_{e}}=5 \mathrm{MeV}$, and $T_{v_{\mu, \tau}}=6 \mathrm{MeV}$. We will discuss the effect of uncertainties in the total neutrino energy and the neutrino temperatures [6, 7]. The $v$-process cross sections are adopted from [10] except charged-current reactions of ${ }^{4} \mathrm{He}$ and ${ }^{12} \mathrm{C}:{ }^{4} \mathrm{He}\left(v_{e}, e^{-} p\right)^{3} \mathrm{He}$, ${ }^{4} \mathrm{He}\left(\bar{v}_{e}, e^{+} n\right){ }^{3} \mathrm{H},{ }^{12} \mathrm{C}\left(v_{e}, e^{-} p\right){ }^{11} \mathrm{C}$, and ${ }^{12} \mathrm{C}\left(\bar{v}_{e}, e^{+} n\right){ }^{11} \mathrm{~B}$. In this study, we focus on the change of the rates of these reactions due to neutrino oscillations because the $v$-process reactions of ${ }^{4} \mathrm{He}$ and ${ }^{12} \mathrm{C}$ are the most important for ${ }^{7} \mathrm{Li}$ and ${ }^{11} \mathrm{~B}$ production. We adopt analytical approximations to the cross sections as a function of energy $\varepsilon_{v}: \sigma_{v}\left(\varepsilon_{v}\right)=\sigma_{0}\left(\varepsilon_{v}-\varepsilon_{t h}\right)^{\alpha}$, where $\varepsilon_{t h}$ is a threshold energy. Coefficients are listed in [7]. We evaluate the rates of the charged-current reactions using the energy dependent neutrino flux, the transition probabilities by neutrino oscillations, and the analytically approximated cross sections (see [7, 11]). 

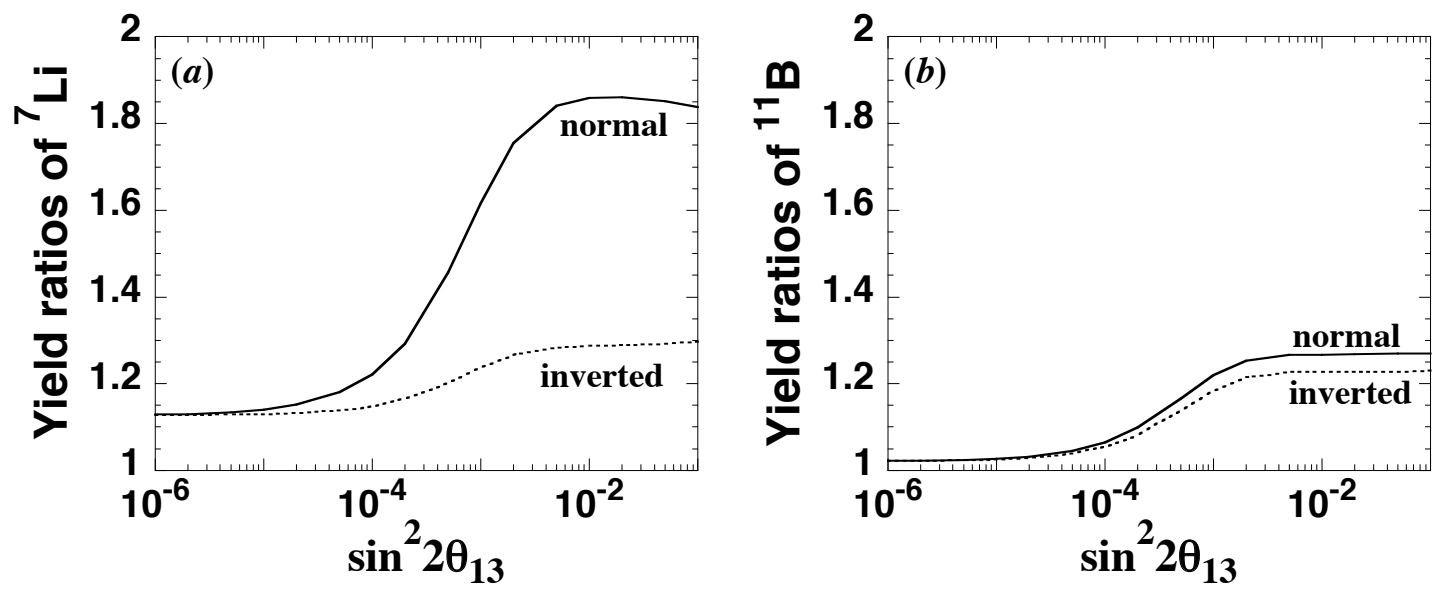

Figure 1: Yield ratios of ${ }^{7} \mathrm{Li}(a)$ and ${ }^{11} \mathrm{~B}(b)$ as a function of $\sin ^{2} 2 \theta_{13}$. Solid lines and dotted lines correspond to a normal mass hierarchy and an inverted mass hierarchy, respectively.

Neutrino oscillation parameters are adopted from recent neutrino experiments. The squaredmass differences $\Delta m_{i j}^{2} \equiv m_{i}^{2}-m_{j}^{2}$ are set to be $\Delta m_{21}^{2}=7.9 \times 10^{-5} \mathrm{eV}^{2}$ and $\Delta m_{31}^{2}= \pm 2.4 \times 10^{-3} \mathrm{eV}^{2}$ where positive and negative values of $\Delta m_{31}^{2}$ correspond to a normal mass hierarchy, $m_{1}<m_{2}<m_{3}$, and an inverted mass hierarchy, $m_{3}<m_{1}<m_{2}$, respectively. The mixing angles $\theta_{i j}$ are set to be $\sin ^{2} 2 \theta_{12}=0.816, \sin ^{2} 2 \theta_{23}=1.0$, and $0 \leq \sin ^{2} 2 \theta_{13} \leq 0.1$. The change of the spectra due to neutrino oscillations is calculated with a Runge-Kutta method and using exact solutions of the oscillations given in [12, 13].

\section{Results}

We demonstrate the effects of neutrino oscillations on the ${ }^{7} \mathrm{Li}$ and ${ }^{11} \mathrm{~B}$ yields in $\mathrm{SN}$ ejecta. Figure 1 shows the ratios of the ${ }^{7} \mathrm{Li}(a)$ and ${ }^{11} \mathrm{~B}(b)$ yields when taking into account of spectral changes by oscillations to those without oscillations. When we do not consider neutrino oscillations, the ${ }^{7} \mathrm{Li}$ and ${ }^{11} \mathrm{~B}$ yields are $2.36 \times 10^{-7} M_{\odot}$ and $6.26 \times 10^{-7} M_{\odot}$.

In a normal mass hierarchy, the ${ }^{7} \mathrm{Li}$ yield increases by about a factor of 1.9 for $\sin ^{2} 2 \theta_{13} \geq 2 \times$ $10^{-3}$. In this range, almost all $v_{e}$ in the He-rich layer are the result of $v_{\mu, \tau}$ conversions. Therefore, the effective neutrino temperature changes from $3.2 \mathrm{MeV}$ to $6 \mathrm{MeV}$. This change in $T_{v_{e}}$ increases the reaction rate of ${ }^{4} \mathrm{He}\left(v_{e}, e^{-} p\right)^{3} \mathrm{He}$, and thus raises the yield of ${ }^{7} \mathrm{Be}$ produced through ${ }^{3} \mathrm{He}(\alpha, \gamma){ }^{7} \mathrm{Be}$. This large $v_{e} \leftrightarrow v_{\mu, \tau}$ exchange is due to the fact that the resonance of mass eigenstates 1 and 3 , i.e., the 13-mixing resonance, is adiabatic. In the case of $\sin ^{2} 2 \theta_{13} \leq 2 \times 10^{-5}$, the ${ }^{7} \mathrm{Li}$ yield is larger by only a small factor of 1.1. This range corresponds to nonadiabatic resonance of the 13-mixing, and flavor exchange is less effective. With increasing $\sin ^{2} 2 \theta_{13}$, the resonance of the 13-mixing changes from nonadiabatic to adiabatic and the ${ }^{7} \mathrm{Li}$ yield increases. There are no resonances of antineutrinos in a normal mass hierarchy. Thus, the increase in the rates of charged-current $\bar{v}_{e}$ reactions is small and does not depend on $\sin ^{2} 2 \theta_{13}$.

In an inverted mass hierarchy, the ${ }^{7} \mathrm{Li}$ yield increases by a factor of 1.3 in the range of $\sin ^{2} 2 \theta_{13} \geq 2 \times 10^{-3}$. In this range, the 13-mixing resonance is adiabatic and, therefore all $\bar{v}_{e}$ 


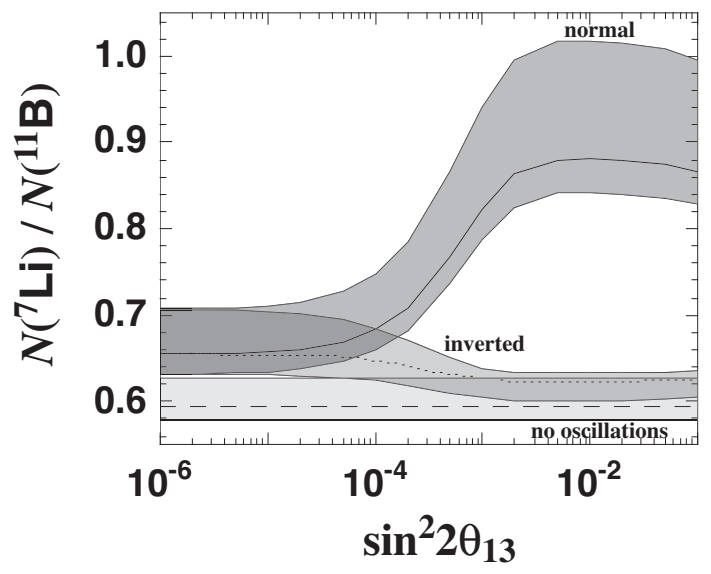

Figure 2: Abundance ratio of ${ }^{7} \mathrm{Li}$ to ${ }^{11} \mathrm{~B}$ as a function of $\sin ^{2} 2 \theta_{13}$. Dark and medium shaded regions correspond to a normal mass hierarchy and an inverted mass hierarchy, respectively. The lightly shaded region is the case where the effect of neutrino oscillations is ignored. Solid, dotted, and dashed lines correspond to the standard neutrino spectra in our study (see the text).

in the He-rich layer result from conversion of $\bar{v}_{\mu, \tau}$. This change raises the temperature $T_{\bar{v}_{e}}$ from 5 $\mathrm{MeV}$ to $6 \mathrm{MeV}$ and the rate of ${ }^{4} \mathrm{He}\left(\bar{v}_{e}, e^{+} n\right)^{3} \mathrm{H}$. However, the difference between $T_{\bar{v}_{e}}$ and $T_{\bar{v}_{\mu, \tau}}$ is smaller than that between $T_{v_{e}}$ and $T_{v_{\mu, \tau}}$, so that the increase in the rate of ${ }^{4} \mathrm{He}\left(\bar{v}_{e}, e^{+} n\right)^{3} \mathrm{H}$ is smaller than that of ${ }^{4} \mathrm{He}\left(v_{e}, e^{-} p\right)^{3} \mathrm{He}$. In $\sin ^{2} 2 \theta_{13} \leq 2 \times 10^{-5}$, the ${ }^{7} \mathrm{Li}$ yield is larger by a factor of 1.1. When the 13-mixing resonance is nonadiabatic, the change of the energy spectra by oscillations does not depend on the mass hierarchies.

The dependence of the ${ }^{11} \mathrm{~B}$ yield on $\sin ^{2} 2 \theta_{13}$ is similar to that of ${ }^{7} \mathrm{Li}$. However, the ${ }^{11} \mathrm{~B}$ yield is larger by a factor of 1.3 even in a normal mass hierarchy and adiabatic 13-mixing resonance. Furthermore, the ${ }^{11} \mathrm{~B}$ yield ratio is insensitive to mass hierarchies. Most of ${ }^{11} \mathrm{~B}$ is produced from ${ }^{4} \mathrm{He}$ by way of ${ }^{7} \mathrm{Li}$ through ${ }^{7} \mathrm{Li}(\alpha, \gamma){ }^{11} \mathrm{~B}$. In a normal mass hierarchy and adiabatic 13 -mixing resonance, the ${ }^{11} \mathrm{~B}$ yield increases by the enhancement of the ${ }^{7} \mathrm{Li}$ yield by way of ${ }^{7} \mathrm{Be}$. However, most of ${ }^{7} \mathrm{Li}$ produced through ${ }^{7} \mathrm{Be}$ does not capture $\alpha$-particles. In an inverted mass hierarchy and adiabatic 13-mixing resonance, the increase in the ${ }^{11} \mathrm{~B}$ yield is due to the enhancement of ${ }^{7} \mathrm{Li}$ production by way of ${ }^{3} \mathrm{H}$. However, small difference of $T_{\bar{v}_{e}}$ and $T_{\bar{v}_{\mu, \tau}}$ makes the enhancement of ${ }^{7} \mathrm{Li}$ and ${ }^{11} \mathrm{~B}$ yields small. A part of ${ }^{11} \mathrm{~B}$ is produced through the $v$-process from ${ }^{12} \mathrm{C}$, especially in the O-rich layers. The reaction rates of ${ }^{12} \mathrm{C}\left(v_{e}, e^{-} p\right){ }^{11} \mathrm{C}$ and ${ }^{12} \mathrm{C}\left(\bar{v}_{e}, e e^{+} n\right){ }^{11} \mathrm{~B}$ increase in the cases of normal and inverted mass hierarchies, respectively. However, the ${ }^{11} \mathrm{~B}$ yield produced from ${ }^{12} \mathrm{C}$ in the He-rich layer is small even with neutrino oscillations.

We have shown in [4] that the ${ }^{7} \mathrm{Li}$ and ${ }^{11} \mathrm{~B}$ yields depend on neutrino temperatures and that we have an uncertainty of a factor of 2.2 in the yields. This factor is larger than the enhancement of the ${ }^{7} \mathrm{Li}$ yield due to neutrino oscillations. Although the temperature dependence is similar among the ${ }^{7} \mathrm{Li}$ and ${ }^{11} \mathrm{~B}$ yields, the enhancement of the yield by neutrino oscillations is different between ${ }^{7} \mathrm{Li}$ and ${ }^{11} \mathrm{~B}$. Thus, we consider the dependence of the ${ }^{7} \mathrm{Li} /{ }^{11} \mathrm{~B}$ abundance ratio on mass hierarchies and $\sin ^{2} 2 \theta_{13}$. Figure 2 shows the ${ }^{7} \mathrm{Li} /{ }^{11} \mathrm{~B}$ abundance ratio with the relation of $\sin ^{2} 2 \theta_{13}$ in normal and inverted mass hierarchies. In this figure, we consider the uncertainties of the total neutrino energy 
and the temperature of $v_{\mu, \tau}$ at the neutrino sphere [6] and the uncertainties of the temperatures of $v_{e}$ and $\bar{v}_{e}$ [7]. When we do not consider neutrino oscillations, the ${ }^{7} \mathrm{Li} /{ }^{11} \mathrm{~B}$ abundance ratio is $0.58-0.63$. In $\sin ^{2} 2 \theta_{13} \leq 1 \times 10^{-5}$, the ${ }^{7} \mathrm{Li} /{ }^{11} \mathrm{~B}$ is $0.63-0.71$. In a normal mass hierarchy and adiabatic 13-mixing resonance $\left(\sin ^{2} 2 \theta_{13} \geq 2 \times 10^{-3}\right)$, the ${ }^{7} \mathrm{Li} /{ }^{11} \mathrm{~B}$ is larger than 0.83 by taking into account uncertainties of neutrino temperatures. Thus, if a large ${ }^{7} \mathrm{Li} /{ }^{11} \mathrm{~B}$ ratio is detected in stars whose atmosphere exhibits a clear signature of a strong contribution of SN ejecta, a normal mass hierarchy and relatively large $\sin ^{2} 2 \theta_{13}\left(2 \times 10^{-3} \leq \sin ^{2} 2 \theta_{13} \leq 0.1\right)$ are suggested. Rebull et al. [14] measured the ${ }^{11} \mathrm{~B} /{ }^{10} \mathrm{~B}$ ratio in HD76932 using the Goddard High-Resolution Spectrograph with the Hubble Space Telescope and inferred a ratio of ${ }^{11} \mathrm{~B} /{ }^{10} \mathrm{~B} \sim 4-10$. Since SNe produce ${ }^{11} \mathrm{~B}$ rather than ${ }^{10} \mathrm{~B}$, stars that exhibit a large ${ }^{11} \mathrm{~B} /{ }^{10} \mathrm{~B}$ ratio may provide direct evidence for the $v$-process. Future observations of ${ }^{7} \mathrm{Li}$ and ${ }^{11} \mathrm{~B}$ abundances in such stars might constrain mass hierarchies and the mixing angle $\theta_{13}$.

There are alternative astrophysical approaches to constraining neutrino mass hierarchies and the mixing angle $\theta_{13}$. One such method involves detection of the diffuse supernova neutrino background (DSNB). Although the DSNB has not yet been detected, improved detection methods and larger detectors may soon lead to the detection of $\bar{v}_{e}$ in the DSNB [15]. In the case of an inverted mass hierarchy and an adiabatic 13-mixing resonance, the $\bar{v}_{e}$ flux significantly differs from what one would find for other oscillation parameters [16, 17, 18]. Another approach involves high redshift galaxy surveys. Since neutrinos with finite masses affect structure formation in the early Universe, future galaxy surveys with higher precision may constrain the total mass of the neutrino mass eigenstates $\left(m_{v, t o t}=m_{1}+m_{2}+m_{3}\right)$ [19, 20]. Combining these approaches even more stringently constrains the mass hierarchy and $\theta_{13}$.

It has been recently discussed the effect on neutrino oscillations from the contribution of neutrino-neutrino interactions in deep region just above a proto-neutron star. In such a deep region, the neutrino flux is so large that the neutrino-neutrino interactions may play an important role in the change of neutrino spectra in addition to the neutrino oscillations. The analysis of the neutrino oscillations with the neutrino-neutrino interactions has been conducted in several limited cases [21, 22], and the effect on the neutrino spectra has been investigated [11]. We would like to investigate the nucleosynthesis including this effect when more definite characteristics of the neutrino-neutrino interactions on neutrino spectra are found quantitatively.

\section{Summary}

We investigated the effects of neutrino oscillations on the ${ }^{7} \mathrm{Li}$ and ${ }^{11} \mathrm{~B}$ production through the $v$-process in a SN model corresponding to SN 1987A. The ${ }^{7} \mathrm{Li}$ and ${ }^{11} \mathrm{~B}$ yields taking into account neutrino oscillations are larger than the corresponding yields without oscillations. In a normal mass hierarchy and $\sin ^{2} 2 \theta_{13} \geq 2 \times 10^{-3}$, corresponding to adiabatic 13 -mixing resonance, the ${ }^{7} \mathrm{Li}$ yield increases by a factor of 1.9. On the other hand, the ${ }^{11} \mathrm{~B}$ yield increases by a factor of 1.3. In other cases of neutrino oscillation parameters, the enhancement of the ${ }^{7} \mathrm{Li}$ and ${ }^{11} \mathrm{~B}$ yields is smaller. In a normal mass hierarchy and $\sin ^{2} 2 \theta_{13} \geq 2 \times 10^{-3}$, the enhancement of ${ }^{7} \mathrm{Li} /{ }^{11} \mathrm{~B}$ is obtained even taking account of uncertainties of neutrino temperatures and the total neutrino energy. Thus, the detection of a ${ }^{7} \mathrm{Li} /{ }^{11} \mathrm{~B}$ enhancement in stars displaying evidence for a definite $\mathrm{SN}$ suggest a normal mass hierarchy and relatively large $\sin ^{2} 2 \theta_{13}$. The answer to the question whether SN neutrino 
nucleosynthesis can constrain neutrino oscillation parameters is affirmative, but the effects are subtle so that one requires high quality abundance measurements for a large sample of stars in order to use this approach in a meaningful way.

\section{References}

[1] R. D. McKeown and P. Vogel, Phys. Rep. 394 (2004) 315.

[2] S. E. Woosley, D. H. Hartmann, R. D. Hoffman, and W. C. Haxton, Astrophys. J. 356 (1990) 272.

[3] T. Yoshida, M. Terasawa, T. Kajino, and K. Sumiyoshi, Astrophys. J. 600 (2004) 204.

[4] T. Yoshida, T. Kajino, and D. H. Hartmann, Phys. Rev. Lett. 94 (2005) 231101.

[5] K. Takahashi, M. Watanabe, K. Sato, and T. Totani, Phys. Rev. D64 (2001) 093004.

[6] T. Yoshida, T. Kajino, H. Yokomakura, K. Kimura, A. Takamura, and D. H. Hartmann, Phys. Rev. Lett. 96 (2006) 091101.

[7] T. Yoshida, T. Kajino, H. Yokomakura, K. Kimura, A. Takamura, and D. H. Hartmann, Astrophys. J. 649 (2006) 319.

[8] T. Shigeyama and K. Nomoto, Astrophys. J. 360 (1990) 242.

[9] T. Shigeyama, K. Nomoto, H. Yamaoka, and F.-K. Thielemann, Astrophys. J. 386 (1992) L13.

[10] R. D. Hoffman and S. E. Woosley, http://www-phys.llnl.gov/Research/RRSN/nu_csbr/neu_rate.html (1992).

[11] A. B. Balantekin and H. Yüksel, New J. Phys. 7 (2005) 51.

[12] K. Kimura, A. Takamura, and H. Yokomakura, Phys. Lett. B537 (2002) 86.

[13] K. Kimura, A. Takamura, and H. Yokomakura, Phys. Rev. D66 (2002) 073005.

[14] L. Rebull, F. Primas, and D. Duncan, in The First Stars, eds. A. Weiss, T. G. Abel, and V. Hill (ESO Astrophysics Symposia; Berlin: Springer), (2000) 176.

[15] C. Lunardini, Phys. Rev. D73 (2006) 083009.

[16] S. Ando, Astrophys. J. 607 (2004) 20.

[17] C. Lunardini, Astropart. Phys. 26 (2006) 190.

[18] H. Yüksel, S. Ando, and J. F. Beacom, Phys. Rev. C74 (2006) 015803.

[19] U. Seljak, et al., Phys. Rev. D71 (2005) 103515.

[20] M. Takada, E. Komatsu, and T. Futamase, Phys. Rev. D73 (2006) 083520.

[21] G. M. Fuller and Y.-Z. Qian, Phys. Rev. D73 (2006) 023004.

[22] A. B. Balantekin and Y. Pehlivan, J. Phys. G 34 (2007) 47. 